\title{
Die Vielfalt der Lebensgemeinschaften
}

\section{Zwischen Gleichstellungsgebot und Diskriminierungsverbot}

\section{Von Wolfgang Lienemann}

Sollen gleichgeschlechtliche Partner eine Ehe schließen können? Sollen gleichgeschlechtliche Paare in derselben Weise wie verschiedengeschlechtliche Paare ein Recht haben, ein Kind oder mehrere Kinder zu adoptieren? Das Ergebnis meiner Überlegungen zu dem mir gestellten Thema ${ }^{1}$ ist auf den ersten Blick ein doppeltes Nein: Ich bin gegen ein (uneingeschränktes) Recht auf Adoption von Kindern durch gleichgeschlechtliche Paare, und ich meine, man soll unter den heutigen Bedingungen eine rechtsförmige Verbindung zweier Frauen oder Männer nicht als "Ehe" bezeichnen. Auf den zweiten Blick aber wird sich das zweite Nein lediglich als ein Zwischenergebnis erweisen, dann ich verbinde damit die Forderung, gleichgeschlechtlichen Partnern eine rechtlich verbindliche Gestalt ihrer Partnerschaft durch das Familienrecht zu eröffnen, welche die meisten Merkmale der heutigen Institution der Ehe umfaßt. Ich plädiere für die gesetzliche Verankerung einer registrierten Partnerschaft im Familienrecht, welche in ihrer Ausgestaltung der Ehe sehr nahekommt, von ihr jedoch in noch näher zu erläuternder Weise auch eindeutig unterschieden bleibt.

Mit dieser These stehe ich unter den theologischen Ethikern im deutschen Sprachraum, die sich zu diesen Fragen bisher geäußert haben, auf den ersten Blick vermutlich ziemlich isoliert da ${ }^{2}$. Mein Plädoyer ist indes, zumindest nach dem Stand meiner derzeitigen Einsicht, lediglich die ausdrückliche Darlegung von zwingenden Konsequenzen, die sich aus einem relativ großen Konsens in Grundfragen der theologischen Sexualethik, des evangelischen Eheverständnisses, der Rechtsethik und der Familienethik ergeben. Insbesondere hinsichtlich der Wahrnehmung und Beurteilung der Homosexualität - ich selbst bevorzuge den Begriff Homophilie - ist es im Bereich der evangelischen Sozialethik in den letzten 25 Jahren zu einer Neubesinnung von Grund auf gekommen ${ }^{3}$, aber gleichzeitig wird immer wieder deutlich, daß es schwer fällt, in den Kirchen wie in der Gesellschaft daraus die möglichen Konsequenzen zu ziehen. Denn eines ist klar: Wenn das Plädoyer für registrierte Partnerschaften ${ }^{4}$ Zustimmung von seiten der Kirchen finden würde, müßte dieses neue Rechtsinstitut natürlich auch für Pfarrerinnen und Pfarrer gelten, es sei denn, auch die evangelischen Kirchen führten den Pflichtzölibat ein. Klar ist aber auch, daß nach wie vor ein ziemlich großer Teil der Kirchenmitglieder erhebliche Mühe hat, homophile Lebensformen und Verhaltensweisen zu akzeptieren. Selbst wo man aufgrund eigener Erfahrungen oder aufgrund rationaler ethischer Überlegungen jede Diskriminierung homophiler Menschen entschieden ablehnt, kann es sehr wohl individuelle, meist emotional getönte Vorbehalte gegen derartige Partnerschaften geben. Trotz weitgehender Toleranzbereitschaft, zumindest in liberalen Kreisen, gegenüber homophilen Neigungen und Lebensformen ist die Ablehnung jeder rechtlichen Institutionalisierung derartiger Partnerschaften nach meinen Beobachtungen in Deutschland und in der Schweiz nach wie vor sehr weit verbreitet.

Zur Begründung und Erläuterung meines Plädoyers sind vorweg einige Unterscheidungen unerläßlich. Ich werde als theologischer Ethiker, nicht als Jurist argumentieren. Dies bedeutet, 
daß ich die Erörterung der Frage, ob und inwieweit aus dem juristischen Verständnis von Personwürde, Selbstbestimmung, Ehe und staatsbürgerlicher Gleichheit im Sinne moderner rechtsstaatlicher Verfassungen die Möglichkeit der Ehe auch für gleichgeschlechtliche Paare gefolgert werden muß, zunächst einmal den Juristen überlasse. Andererseits können die Juristen jedoch nicht willkürlich davon absehen, welche kulturellen Überlieferungen etwa das Verständnis der Ehe nicht nur in der europäischen Geschichte geprägt haben, sondern eben auch in der Gegenwart von sehr vielen Menschen aufgrund ihrer sittlichen und religiösen Überzeugungen geteilt und anerkannt werden. Gleichzeitig muß man als theologischer Ethiker genau unterschieden zwischen Institutionen und Normen, die für alle Menschen Geltung beanspruchen können sollen, und solchen, die bei den Gliedern der Gemeinschaft der Kirche Anerkennung finden. Anders gesagt: der Umgang mit Fragen der Sexualität, der Ehe und Familie im Staate kann und darf nicht umstandslos dem kirchlichen Selbstverständnis folgen, und die kirchliche Lehre in diesen Bereichen muß sorgfältig unterscheiden zwischen Ratschlägen und Forderungen, die das allgemeine Recht für alle Bürger betreffen, und solchen Regeln und Ordnungen, die sich nur auf die Glieder der Kirche erstrecken. Am Beispiel der Ehe läßt sich diese Unterscheidung leicht erläutern: staatliches Eherecht muß, wo es die Ehescheidung ermöglicht, auch die Wiederverheiratung ermöglichen und regeln; die Kirche kann indes nicht vom Staat gezwungen werden, Geschiedene wieder zu trauen, sondern sie tut dies nur, wo sie aus Gründen ihrer eigenen Lehre überzeugt sein kann, damit das Richtige zu tun, das heißt, dem Willen Gottes zu entsprechen. - Für unser Thema sind derartige Unterscheidungen wichtig, denn es kann nicht von vornherein ausgemacht sein, ob eine kirchliche Empfehlung an den Gesetzgeber zugunsten von registrierten gleichgeschlechtlichen Partnerschaften im weltlichen Recht auch eine entsprechende Form einer kirchlichen Trauung nach sich ziehen müßte (wenngleich das möglich, ja, wahrscheinlich wäre); und ebensowenig kann sich die Kirche durch die Nichteinführung derartiger Bestimmungen in das staatliche Familienrecht daran hindern lassen, liturgische Segenshandlungen für homophile Paare zu vollziehen, wenn ihr dies aufgrund theologischer Einsichten als geboten unabweisbar wird.

Eine theologische Urteilsbildung in diesem Grenzbereich von staatlichem und kirchlichem Recht, allgemeiner Rechtsethik und kirchenspezifischen Urteilsgründen wird sinnvollerweise zuerst zu fragen haben, wie eine heutige theologisch-ethische Beurteilung der Homophilie aussehen kann (I). Sie wird dann in einem zweiten Schritt die Folgerungen entwickeln müssen, die sich daraus für die verbindliche Gestaltung von Lebensgemeinschaften, insbesondere für die Ehe, ergeben (II). In einem dritten Schritt sollen dann einige Konsequenzen sowohl im Blick auf kirchliche Handlungsmöglichkeiten und Ordnungsformen als auch hinsichtlich rechtspolitischer Erwägungen skizziert werden (III).

\section{Zur theologisch-ethischen Beurteilung der Homophilie}

\section{Erfahrungen im Umgang mit homophilen Menschen}

Bei Gesprächen, Stellungnahmen oder Leserbriefen von Christenmenschen zur Frage der Homophilie habe ich immer wieder zwei Beobachtungen gemacht: Erstens verweisen 
insbesondere jene, die alle Formen gelebter Homophilie verwerfen, auf jene bekannten Stellen des Alten und Neuen Testamentes, in denen von Homosexualität als einem gräßlichen Laster die Rede ist. Diese Stellungnahmen kommen vor allem von Menschen, die die Botschaft der Bibel wortwörtlich ernst zu nehmen bemüht sind. Ich nenne sie mit Absicht nicht "Fundamentalisten«, sondern bibeltreue Menschen, obwohl sicher unter ihnen auch solche begegnen, die aus der frohen Botschaft ein autoritäres Gesetz machen, das die Gewissen knechtet und zur Ursache vieler psychischer Leiden werden kann. Aber zuerst einmal achte ich alle, die in dem Sinne »bibeltreu « genannt werden können, daß sie dem Hören auf das Zeugnis der Bibel immer eine höhere Würde, einen größeren Reichtum und eine menschenfreundlichere Autorität zutrauen als ihren eigenen Wünschen und Bedürfnissen. Sie lassen die Bibel auch gegen sich selbst gelten. Ihre Schriftauslegung verdient deshalb auch in der Frage der Homophilie Gehör, freilich kein Monopol.

Zweitens habe ich beobachtet, daß das individuelle Urteil über Homophilie ganz entscheidend davon geprägt ist, ob jemand persönlich homosexuell orientierte Frauen oder Männer kennt, vielleicht mit ihnen befreundet ist, sie vielleicht in der eigenen näheren oder ferneren Verwandtschaft hat. Insbesondere wenn eigene Kinder sich als homophil veranlagt offenbaren, bricht für manche Eltern eine Welt zusammen. Sofern sie dann jedoch sich ein Herz fassen und mit den Problemen Homosexueller auseinandersetzen, auch deren Partnerinnen oder Partner kennenlernen, pflegt sich sehr häufig das Urteil zu ändern. Und dabei ist mein Eindruck, daß in dem Maße, wie der erste Schock einer neuen Zuwendung zur Person des Homosexuellen weicht, die anfängliche Fixierung auf die besondere Ausprägung der Sexualität wieder zurücktritt - wie man ja auch heterosexuell orientierte Menschen im allgemeinen nicht zuerst und ausschließlich als potentielle Sexualpartner wahrnimmt, sondern als Spielkameraden, Polizistinnen, Busfahrer oder Pfarrerinnen, die mehr oder weniger aufregend oder anziehend sein können. Ich will sagen: Es gehört zu unseren spezifisch menschlichen Möglichkeiten, die Andere oder den Anderen nicht primär unter dem Aspekt des Sexuellen wahrzunehmen, sondern als eine in sich reiche Gestalt mit vielen Eigenschaften und Kräften, zu denen freilich auch meist besonders mächtig die Sexualität gehört. Menschlich aber ist deren Einordnung in den Kreis der anderen Eigenschaften und Vermögen eines Menschen, und wo dies im Leben mit Homosexuellen geschieht, verliert der sexuelle Aspekt ihrer Existenz meist von selbst seine als bedrohlich empfundene Wirkung auf andere Menschen. Und dies wiederum ist eine gute Voraussetzung dafür, sich gemeinsam den biblischen Überlieferungen zuzuwenden - nicht, um diese nach eigenem Geschmack und Interesse umzubiegen, sondern um mir von ihnen etwas sagen zu lassen, das ich sonst von mir selbst her nicht weiß.

\section{Zum biblischen Befund}

Der biblische Befund ${ }^{5}$ ist in erster Annäherung einfach und klar. Sowohl das Alte wie das Neue Testament lehnen homosexuelles Verhalten strikt ab. Die entsprechenden Belege sind bekannt und werden regelmäßig zitiert, etwa aus dem Heiligkeitsgesetz, wo es heißt: »Du sollst nicht bei einem Mann liegen, wie man bei einer Frau liegt. Das wäre ein Greuel« (Lev 18,22; vgl. 20,13). 
Homosexualität wird insbesondere in Texten verdammt, die in ihrem weiteren Zusammenhang die kultischen Reinheitsforderungen Israels in scharfer Abgrenzung gegenüber kanaanäischen Fremdkulten geltend machen, die vor allem aber eindeutig tabuisieren, was in einer antiken patriarchalischen Agrargesellschaft wie dem alten Israel einfach als indiskutabel galt ${ }^{6}$. Unter den neutestamentlichen Stellen (Röm 1,26.f; 1 Kor 6,9f.; 1 Tim 1,9f.) sind besonders jene drastischen Wendungen geläufig, die Paulus im ersten Kapitel des Römerbriefes geprägt hat: die Verkehrung des natürlichen in einen unnatürlichen Umgang der Menschen miteinander ist selbst schon die Strafe, die aus dem Zerbrechen der Gottesbeziehung herrührt.

Bei genauerer Lektüre ergeben sich aus dem biblischen Befund jedoch Einsichten, die weit über die scheinbar eindeutigen Verdammungsworte hinausgehen. Erstens ist erstaunlich, daß insgesamt gesehen in der ganzen Bibel von Homosexualität nur sehr selten die Rede ist. Zweitens stehen insbesondere die alttestamentlichen Belege zu einem großen Teil im Kontext der Abwehr von Verhaltensweisen, die geeignet sind, das soziale und sexuelle Gefüge der Großfamilie zu zerstören. Drittens ist Homosexualität im Neuen Testament ein ausgesprochenes Randthema; die Jesustradition spricht überhaupt nicht davon, auch die Synoptiker und die johanneische Tradition schweigen dazu. Nur dort, wo die städtischen Missionsgemeinden in hellenistischer Umwelt angesprochen sind, wird Homosexualität zum Thema. Viertens haben diese wenigen neutestamentlichen Belege ausschließlich christliche Gemeinden zum Adressaten; deren sexualethische Grundsätze mit staatlicher Hilfe öffentlich durchsetzen zu wollen, lag indessen gänzlich außerhalb aller Vorstellungen.

Es wäre nun aber ein ganz unsachgemäßer Umgang mit der Bibel, aus dieser Randständigkeit des Themas seine Belanglosigkeit zu folgern. Das wäre Interessenhermeneutik in dem Sinne, daß man an einem fremden Wort nur zur Kenntnis nimmt, was man schon vorab als für einen selbst einleuchtend, richtig und gut betrachtet. Sachlich angemessen ist dagegen nur eine Deutung, die erstens die wenigen Urteile über Homosexualität in den Zusammenhang der sonstigen Äußerungen eines biblischen Autors einordnet, und die zweitens sich zutraut, auch diesen Autor oder diese Überlieferung daran zu messen, was als Zentrum, Zentralperspektive oder unverlierbarer Gehalt des Evangeliums standhält. Mit anderen Worten: wir müssen immer wieder den Mut haben, jede einzelne biblische Aussage und Überlieferung daran zu messen, was dem Gesamtzeugnis der Bibel und dessen einzigartiger Konzentration in der Christusüberlieferung Ausdruck gibt.

\section{Sachkritik biblischer Traditionen}

Diese Aufgabe der Sachkritik biblischer Traditionen? betrifft auch die wirkungsmächtigen Behauptungen des Paulus über die menschliche Sexualität ${ }^{8}$. Zurückhaltend geurteilt gilt, daß für ihn die Sexualität der Menschen nicht eine gute Gabe Gottes, sondern vor allem eine satanische Versuchung ist. Die Ehe erscheint in erster Linie als ein notwendiges Übel, um die Macht der sexuellen Wünsche einigermaßen ehrbar zu kanalisieren. Besser ist es, angesichts der bevorstehenden Wiederkunft Christi und des baldigen Endes der Welt unverheiratet zu bleiben; wer aber diese Gabe nicht besitzt, soll, um nicht der Sünde völlig zu erliegen, sich verehelichen. Der Christentumskritiker Joachim Kahl hat diese Auffassung aggressiv, aber nicht unzutreffend, so 
umschrieben: "Die Ehe ist für Paulus das freudlose Bordell in der eigenen Wohnung für diejenigen, die nicht enthaltsam leben können. $\ll^{9}$

Ich sehe zwei Linien, auf denen sich gleichwohl bei Paulus die Einsicht in den lebensfördernden Willen des Evangeliums Bahn bricht. Es handelt sich erstens um seine Beurteilung der Phänomene der Leiblichkeit, zweitens um seine Hochschätzung der Liebe (agape). Paulus hat nämlich an die Mitglieder der Gemeinde zu Korinth geschrieben, daß jede und jeder von ihnen in ihrer Leiblichkeit Glieder Christi darstellen. Die Leiber der Menschen sind Paulus zufolge Tempel des heiligen Geistes (1 Kor 6,19), Tempel freilich, die von Grund auf entweiht werden können. Ich denke nun, daß derartige Äußerungen nicht einfach nur Ausdruck eines Sexualpessimismus sind, sondern darauf aufmerksam machen wollen, daß Menschen für ihre Leiblichkeit und deren Darstellungs- und Handlungsformen verantwortlich sind. Es geht dabei, mit Kant zu reden, um Pflichten gegen sich selbst und gegen andere. Wir existieren immer nur als Geist und Seele eines, unseres Leibes in der uns zugemessenen Zeit unserer Vergänglichkeit; in dieser Zeit sind wir für unsere leibhaftigen Vollzüge verantwortlich.

Woran aber soll sich diese Verantwortlichkeit orientieren? Es gibt nur eine durchgehende Antwort auf diese Frage bei Paulus, und die lautet, daß alles, was Menschen tun, »in der Liebe geschehen « soll (1 Kor 16,14). Die Liebe ist des Gesetzes Erfüllung; die Liebe ist Maßstab des Redens - insbesondere der theologischen Rede in der Gemeinde -, des diakonischen Helfens und Teilens, der Weissagung und aller Erkenntnis. Alles menschliche Tun ohne die Liebe wäre »nichts nütze« (1 Kor 13,3). Aber diese so eindeutig klare Zentralantwort hat Paulus nicht mit seiner Sicht der Sexualität zu verbinden vermocht; er hat diese gleichsam von jener abgespalten und zu verdrängen versucht, und für Jahrhunderte sind ihm viele Theologen darin gefolgt, daß sie dem sinnlich-menschlichen Eros die himmlisch-unsinnliche Agape gegenübergestellt haben ${ }^{10}$. Fragt man aber nach der Einheit des biblischen Zeugnisses, so geht es doch darum, wie Sexualität und Erotik in ihrem sinnlichen und geistigen Reichtum von der Liebe geformt werden können. Es geht, wenn man Paulus gegen seinen eigenen Sexualpessimismus verteidigt, darum, »die Liebe, die dem Christen durch den Glauben verheißen und befohlen ist, und die Liebe, die mit seiner Sexualität zusammenhängt, zu versöhnen «"11.

Sachkritik an Paulus bedeutet demzufolge, seine Äußerungen zu Sexualität und Ehe unter dem von ihm selbst als grundlegend eingeführten Kriterium der Liebe zu prüfen. In dieser Perspektive hat Walter Neidhart einmal phantasievoll ausgemalt, wie das paulinische Hohelied der Liebe lauten könnte, wenn der Apostel selbst befriedigende Sexualität gekannt hätte; es hätte dann vielleicht so geklungen ${ }^{12}$ :

Die Liebe ist langmütig, die Liebe ist gütig,

die Liebe wartet darauf, erwidert zu werden.

Sie eifert nicht, sie prahlt nicht, sie bläst sich nicht auf,

und sie ist dankbar für die Gegenliebe des andern

und ist stolz, wenn sie Konflikte überwindet.

Die Liebe tut nichts Unschickliches,

aber freut sich im Kämmerlein am Spiel der Zärtlichkeit

und am schönen Leib des andern.

Die Liebe sucht nicht ihren Vorteil,

aber bejaht die eigene Bedürftigkeit 
und ist fähig, vom andern Liebe und Hilfe zu empfangen.

Sie läßt sich nicht zum Zorn reizen, sie trägt das Böse nicht nach,

aber sie leidet an der Bosheit des andern

und ist froh, wenn auch er die Sonne nicht über seinem

Zorn untergehen läßt.

Sei freut sich nicht über das Unrecht, sondern freut sich

über die Wahrheit,

sie ist nicht traurig über Spannungen mit dem andern,

aber trauert, wenn der Mut fehlt, diese zur Sprache

zu bringen.

Sie trägt - nicht alles. Sie glaubt - nicht alles.

Sie hofft - nicht alles. Sie hält - nicht allem stand.

Aber sie trägt vieles und glaubt vieles und hofft vieles.

Und wenn sie sich weigert zu tragen, wenn sie zum andern

nein sagt, erfährt sie darin den Wert der eigenen Person.

Dieser Wert ist nicht preiszugeben, wenn der andere einen vollwertigen

Partner haben soll.

Die Transformation des Hohenliedes habe ich zitiert, weil sie bei dem Versuch, die Versöhnung von Sexualität und Liebe gegen Paulus mit Paulus zur Sprache zu bringen, ganz selbstverständlich zu Formulierungen greift, welche hetero- wie homosexuellen Erfahrungen Ausdruck zu verleihen vermögen. Im Mittelpunkt steht die Anerkennung des Anderen als eines einmaligen geliebten $\mathrm{Du}$, und zugleich ist von der Angewiesenheit jedes Ich auf ein Gegenüber die Rede, welches als ein Anderes seinerseits das einmalige Ich bejaht, anerkennt und begehrt. In der Perspektive von 1 Kor 13 ist diese Gestalt der Liebe (agape) die umfassende Wirklichkeit, der sich alles andere einordnet, und zwar - über Paulus hinaus - eben nicht nur die Erkenntnis, sondern auch die Erotik und die Sexualität.

\section{Verantwortung für die Leiblichkeit}

Habe ich mit dieser Interpretation und Kritik nun doch Interessenhermeneutik betrieben, um aus Texten herauslesen zu können, was in mein Konzept paßt, um nicht hören zu müssen, was meinen Vorurteilen und Wünschen widerspricht? Ich denke nicht. Denn es ist ganz unbestreitbar, daß selbst für den geradezu pathologisch sexfeindlichen Paulus die Sexualität kein Ober-, sondern eben - ein Nebenthema ist. Dieses Thema steht, wie alle Themen bei Paulus, in der unendlich weiten Perspektive, wie wir mit allen unseren Gaben und Fähigkeiten Gott und dem konkreten Mitmenschen als Frau und Mann zu dienen vermögen. Dreht man diese Reihenfolge um, wird alles verkehrt, buchstäblich pervers. Dann wird die Sexualität erst verselbständigt, von uns als Personen abgespalten, verdrängt oder glorifiziert, und dann hat unser eigenes Verhältnis zu unserer und unserer Nächsten Leiblichkeit nichts mehr mit dem Willen Gottes zu tun. Es ist demgegenüber ganz im Sinne des Paulus gedacht, wenn wir danach fragen, ob und wie unsere natürliche Leiblichkeit und unsere individuell immer ganz besondere sexuelle Prägung so zu einem Teil unserer ganzen Person werden können, daß der 
Leib zu einem "Tempel des heiligen Geistes « $(1$ Kor 6,19$)$ wird ${ }^{13}$. Dieses merkwürdige Bild mahnt uns, für unsere eigene Leiblichkeit und die unserer Nächsten besorgt zu sein - von der Ernährung und dem Gebrauch von Genußmitteln und Drogen bis hin zur Gabe der Sexualität. Leiblichkeit ist nach dem schönen Worte Oetingers das Ende der Wege Gottes. Weil in diesem Sinne die Sexualität zu den natürlichen Gaben in Gottes guter Schöpfung gehört, welche wir durch unseren Gebrauch ebenso entweihen wie heiligen können, gilt dies nach meiner Überzeugung auch für jene Ausprägung der Sexualität, die ihren Ausdruck in der Homophilie findet.

\section{Zwischenergebnis}

Aus diesen kurzen Überlegungen ergibt sich eine erste Zwischenbilanz: in evangelischer Perspektive ist alle Sexualität zuerst und entscheidend auf Sozialität angelegt. Sie ist Teil elementarer menschlicher Beziehungen. Sie ist natürlich zuerst Ausdruck menschlicher Selbstbestimmung, aber diese soll in einer Weise Gestalt finden, daß sie zugleich die Personalität der oder des Anderen anerkennt, achtet und dieser gegenüber dienstbar ist. Alle diese Bestimmungen, so denke ich und so verstehe ich insbesondere Selbstzeugnisse von Schwulen und Lesben ${ }^{14}$, gelten für alle Menschen. Hinsichtlich der lebenslangen Aufgabe, die individuelle Sexualität als Teil der eigenen Person und Lebensführung zu integrieren und zugleich als Teil der gemeinsamen Lebensführung mit einer Partnerin oder einem Partner zu gestalten, sind hetero- wie homosexuell geprägte Menschen wesentlich gleich. Wesentlich Gleiches darf man aber nicht ungleich behandeln, ohne negativ zu diskrimieren; dies ist jedenfalls ein eherner Grundsatz neuzeitlichen Rechtsverständnisses, dem die Kirchen ihre Zustimmung nicht verweigern können ${ }^{15}$.

\section{Kriterien und Gestalten verbindlicher Lebensgemeinschaften}

\section{Ehe als Institution}

Menschliche Gestaltung der Sexualität ist unreglementierbar, anarchisch frei und -im Fall des Gelingens - phantasievoll und im wörtlichen Sinne kreativ. Wenn irgendwo gilt hier die paulinische Maxime des »alles ist mir erlaubt «, aber Paulus hat auch hinzugefügt, daß nicht alles nützlich ist und gut tut und nichts den Menschen gefangen nehmen und abhängig machen soll $(1$ Kor 6,12). Hier zu unterscheiden ist schwer, und darum ist die Gratwanderung zwischen der unaufgebbaren Freiheit und dem, was mir und der oder dem Anderen gut tut, von Abstürzen bedroht. In dieser Gefährdung existieren ganz offensichtlich alle Menschen, unabhängig von ihrer besonderen sexuellen Prägung.

Die Gefahr liegt vor allem in der Verfehlung und Verletzung der oder des Anderen. Sexualität, die mit der Liebe versöhnt ist, vereinigt daher die individuelle Selbstbestimmung mit der Wahrnehmung, Anerkennung und Achtung der oder des Anderen. Diese Wahrnehmung, Anerkennung und Achtung stellen aber keinen isolierten Akt dar, sondern eine 
Geschichte von Begegnungen, mit Höhen und Tiefen, Schweigen und Sprechen, Schuld und Verzeihung. Es ist eben eine menschliche Geschichte. Und weil wir Menschen immer in gewisser, allerdings unterschiedlicher Weise hilfsbedürftig bleiben, damit unser Leben gelingen kann, bedürfen wir etlicher Formen und Regeln, die uns bei der Gestaltung dieses Weges helfen und entlasten. Diese Formen und Regeln sind wohl dann am hilfreichsten, wenn sie in der Gemeinschaft, der wir angehören, mit großer Selbstverständlichkeit beachtet werden; wir müssen dann nicht alle Räder, die das Leben bewegen helfen, ständig neu erfinden.

In diesem elementaren Sinne nenne ich die Ehe eine Institution. Martin Luther hat bekanntlich die Ehe »ein weltlich Ding " genannt, aber der Sinn, den er damit verband, wird meist verfehlt. Denn für ihn war die Ehe eine göttliche Institution (eine institutio beziehungsweise ordinatio $\mathrm{Dei})^{16}$. Was aber ist eine Institution? Wir verbinden damit heute meist Assoziationen wie Organisation und Bürokratie. Nach 1945 hat es, wenigstens in Deutschland, eine breite rechtstheologische Erörterung zum Verständnis des Sinns von Institutionen gegeben ${ }^{17}$. Dabei hat man drei Merkmale einer Institution wie der Kirche, des Staates oder eben auch der Ehe unterschieden: (1) Das Moment einer göttlichen Stiftung, (2) typische, auch in verschiedenen Zeiten und Kulturen wiederkehrende Grundstrukturen, sowie (3) die Möglichkeit menschlicher Gestaltung und Entscheidung im Umgang mit den zwei ersten Merkmalen. Institutionen können insofern verstanden werden als "soziale Daseinsstrukturen der geschaffenen Welt als Einladung Gottes zu ordnender und gestaltender Tat in der Freiheit des Glaubensgehorsams gegen sein Gebot «18. Auch wenn diese Redensweise etwas altertümlich klingen mag, weist sie darauf hin, daß Institutionen nicht einfach als eine fertige Ordnung oder ein Gehäuse vorgegeben sind, in welche man sich einzufügen hat, sondern sie sollen als Mittel und Hilfen zu einer gelingenden Lebensführung erkannt, übernommen und frei ausgestaltet werden. In diesem Sinne steht die Ehe zur Freiheit der menschlichen Selbstbestimmung nicht im Widerspruch, sondern im Verhätlnis eines durch kulturelle Überlieferungen geprägten Angebotes zu verantwortlicher Gestaltung von Sexualität und Liebe. Erst die Liebe, dann, wenn schon, die Ehe! Ich folge hier gern der sonst, besonders im Blick auf die Homosexualität, hochproblematischen Lehre Karl Barths, der schreibt: "Ehe als Lebensgemeinschaft ist also die Bewährung der Liebe. In der Ehe als Lebensgemeinschaft geht es nämlich darum, das Ja der Liebe im Ernstfall zu wiederholen. (...) >Im Ernstfalk heißt: eben das Alles in der Folge von unabsehbar vielen Tagen zu je 24 Stunden, von unabsehbar vielen Jahren zu je 52 Wochen - und eben das in der auf beiden Seiten Alles aufdeckenden Intimität eines alltäglichen - sehr alltäglichen! - und auch allnächtlichen Zusammenseins, in welchem man sich bald genug erschreckend genau kennenlernt, in welchem das Größte erstaunlich klein, aber auch das Kleinste erstaunlich groß werden kann. IIm Ernstfalk heißt: ein Kollektiv, ein Wir, ein Paar geworden sein und nun als solches existieren - nach außen nicht nur, sondern, was die Sache auch nach außen allein möglich macht, nach innen, und in den gegenseitigen Beziehungen nicht nur, sondern im Denken, Wollen und Fühlen beider Beteiligter, auf dem diese Beziehungen beruhen müssen, wenn sie haltbar sein sollen. Dieser Ernstfall der Liebe ist die Ehe als Lebensgemeinschaft. «19

Als Ernstfall dieser Lebensgemeinschaft ist die Ehe konkret Wohn-, Wirtschafts- und Geschlechtsgemeinschaft. Als Geschlechtsgemeinschaft hat sie von Natur her die Aufgabe und 
Möglichkeit, zur Reproduktion der menschlichen Gattung beizutragen; nicht jede einzelne eheliche Lebensgemeinschaft, aber die weitaus größte Zahl von ihnen enthält in sich diesen Naturzweck. Als Geschlechtsgemeinschaft ist sie zugleich Ort und Schauplatz außerordentlicher Intimität; als Sexualwesen sind Menschen ja nicht nur physisch nackt, sondern auch mehr oder weniger als Personen unverborgen. Das bedeutet, daß die Geschlechtsgemeinschaft auch der Ort unserer größten Verletzbarkeiten ist. Aus diesem Grunde bedürfen wir alle und ganz besonders diejenigen, die in einer Beziehung die Schwächeren sind, gerade hier des Schutzes. Diesen will besonders die immer noch als "bürgerlich" gescholtene rechtlich verbindliche, auf Dauer angelegte Ehe bieten: wir nennen sie »bürgerlich«, nicht weil sie den Mief der Kleinbürgerlichkeit des "bourgeois" verbreiten soll, sondern weil sie auch und gerade in der Intimität dem Schutz jeder Person und der Freiheit des »citoyen« oder der »citoyenne« dienen will. Insofern war die viel kritisierte Vertragstheorie der Ehe, wie sie Kant vertreten hat ${ }^{20}$, ein Beitrag zum Schutz und zur Emanzipation des nicht nur im 18. Jahrhundert schwächeren Partners in einer Lebensgemeinschaft.

\section{Ehe für alle?}

Die Frage lautet nun: Darf und soll diese Institution mit ihren entlastenden, hilfreichen und schützenden Funktionen nur verschiedengeschlechtlichen Partnern zugänglich sein, oder muß man sie auch gleichgeschlechtlichen Partnern öffnen? Wenn es keine überzeugenden Gründe rechtlicher, medizinischer, politischer, philosophischer oder theologischer Art dagegen gibt, muß man diese Öffnung vollziehen, wenn sie verlangt wird, weil andernfalls eine zu Recht beanspruchte Gleichbehandlung willkürlich verweigert würde.

Die Ungleichbehandlung gleichgeschlechtlicher Partner gegenüber Eheleuten im heutigen Recht auch der meisten Rechtsstaaten ist bekannt, aber es gibt seit 1989 in Dänemark und neuestens auch in Norwegen und Schweden vielbeachtete Beispiele einer zunehmenden Gleichbehandlung ${ }^{21}$. (Diese Tatsache kann künftig übrigens auch rechtliche Auswirkungen auf Staaten haben, die am bisherigen Eherecht von sich aus nichts ändern wollen, heißt es doch beispielsweise im Artikel 54, Absatz 3 der Schweizer Bundesverfassung: "Die in einem Kanton oder im Auslande nach der dort geltenden Gesetzgebung abgeschlossene Ehe soll im Gebiete der Eidgenossenschaft als Ehe anerkannt weden.«) Homophile Menschen beklagen sich insbesondere darüber, daß die Partner nicht den rechtlichen Status von »Angehörigen« haben: sie haben kein gegenseitiges Zeugnis- und Auskunftsverweigerungsrecht, bei Unglück, Krankheit und Tod muß der gesunde Partner immer wieder hinter den leiblichen Angehörigen zurücktreten, im Bereich des Justizvollzuges haben die gleichgeschlechtlichen Partner kein Besuchsrecht. Hinzu kommen dann die vielen Ungleichbehandlungen mit teilweise einschneidenden wirtschaftlichen Folgen im Steuer-, Unterhalts-, Miet-, Haftungs- und Rentenrecht, die ich hier nicht aufzählen muß, bis hin zu den ausländerrechtlichen Diskriminierungen gleichgeschlechtlicher Partner ${ }^{22}$. Nur bei wenigen Herkunftsländern wie dem Iran ist in Deutschland die homosexuelle Prägung eines Asylbewerbers ein Grund für die Asylgewährung, weil ihm im Heimatland Verfolgung, Folter und Tod drohen.

Ich denke, daß man in etlichen europäischen Staaten die allzu offenkundigen Diskriminie- 
rungen gleichgeschlechtlicher Lebenspartner in den kommenden Jahren abbauen wird, und sei es nur, daß man die Möglichkeiten privatrechtlicher Vereinbarungen in derartigen Fällen erweitert und verbessert, so daß wenigstens in materieller und statusrechtlicher Hinsicht mehr Gleichbehandlung angestrebt wird. Aber damit werden sich homophile Menschen sicher nicht zufrieden geben. Sie argumentieren in Deutschland etwa folgendermaßen: (1) Der Begriff der Ehe ist gesetzlich nicht abschließend definiert. (2) Die grundrechtliche Garantie der Ehe nach Artikel 6 GG umfaßt nicht nur das Recht oder die Freiheit, eine Ehe überhaupt eingehen zu können, sondern die Ehe mit einem selbstgewählten Partner aufgrund gemeinsamen Konsenses. (3) Während nach herkömmlichen "christlich-abendländischen Bild der Ehe« nur verschiedengeschlechtliche Partner die Ehe eingehen konnten und Homosexualität als unsittlich und strafwürdig galt, hat sich seit einiger Zeit ein grundlegender Wandel der Auffassungen von Sexualität und Ehe vollzogen, so daß eine Diskriminierung von Menschen aufgrund ihrer sexuellen Prägung dem Gleichheitsgebot von Artikel 3 Absatz 3 GG widerspricht. Daraus folgt, wie der deutsche Bundesanwalt Manfred Bruns argumentiert: "Wenn aber gleichgeschlechtliche Lebensgemeinschaften nach heutiger Auffassung weder strafbar noch unsittlich sind, stellt sich die Frage, ob das Eheverbot für gleichgeschlechtliche Partnerschaften tatsächlich noch mit dem Grundrecht der Eheschließungsfreiheit zu vereinbaren ist. (...) Zumindest muß gleichgeschlechtlichen Paaren die Möglichkeit eröffnet werden, ihre Partnerschaften in ähnlicher Weise rechtlich abzusichern, wie das verschiedengeschlechtlichen Paaren durch die Eheschließung möglich ist. $\ll^{23}$

Es geht also ausdrücklich um die rechtliche Absicherung einer Partnerschaft, um Schutz und Förderung einer monogamen, auf Dauer angelegten Lebensgemeinschaft. Man sollte das nicht mit dem Vorwurf abtun oder bagatellisieren, da $B$ dahinter auch materielle Interessen an einer Gleichbehandlung stehen, zumal derartige Interessen zweifelsohne auch den Entschluß zur Ehe mitbestimmen können. Vielmehr muß man zuerst einmal zur Kenntnis nehmen, daß homophile Menschen tatsächlich eine Ehe eingehen wollen, daß heißt eine Institution dauerhafter Lebensgemeinschaft und wechselseitiger Verantwortlichkeit übernehmen und gestalten wollen, die als solche weltlicher Art ist, in der aber Christenmenschen zugleich eine Anordnung Gottes erkennen. Die Frage lautet jetzt: Darf der freie Zugang zu dieser Institution Menschen verwehrt werden?

\section{Argumente gegen eine Gleichstellung unterschiedlicher Lebensgemeinschaften}

Die wichtigsten Argumente gegen eine Gleichstellung von gleich- und verschiedengeschlechtlichen Lebensgemeinschaften im Rahmen des Rechtsinstituts der Ehe hat Hermann Ringeling schon vor Jahren kritisch erörtert. Seine Argumente verdienen auch deshalb nach wie vor Gehör ${ }^{24}$, weil er ein Pionier auf dem Wege zum Abbau sexueller Diskriminierungen in Kirche und Gesellschaft war. Weltoffene Liberalität und ein Engagement für die lebensfreundlichen und therapeutischen Dimensionen des christlichen Glaubens verbinden sich in seinen Schriften. Trotzdem vermag ich seine Einwände gegen die Gleichstellungsforderung nicht zu teilen. Seine wichtigsten Argumente ${ }^{25}$ betreffen (1) die Tatsache oder Vermutung eines relativ häufigen Partnerwechsels besonders unter homophilen Männern, (2) die Annahme, daß rechtspolitische 
Forderungen von Homosexuellen oft nur Mittel zur Veränderung der Gesamtgesellschaft sind, (3) die Erwägung, daß die Ablösung der Sexualität von der Fortpflanzungsfunktion und die Konzentration auf eine Partnerin oder einen Partner des eigenen Geschlechtes eine eigentümliche »autistische Schwäche« (Pfürtner ${ }^{26}$ ) zum Ausdruck bringe, sowie (4) die These, daß die Gleichstellungsabsicht hinsichtlich der homophilen Lebenswirklichkeit darauf hinauslaufe, die Ehe als Inbegriff einer vorbehaltlosen, auf Dauer angelegten monogamen Lebensgemeinschaft aufzuheben.

Die ersten zwei Argumente, zuerst veröffentlicht 1987, spielen, wenn ich recht sehe, heute wohl keine Rolle mehr. Für das dritte Argument muß ich mich als inkompetent erklären, sehe aber, $\mathrm{da} ß$ die damit zusammenhängenden Fragen Aufmerksamkeit verdienen. Beim vierten Argument liegt mein eigentlicher Differenzpunkt zu Hermann Ringeling und dem ganz ähnlich argumentierenden Trutz Rendtorff ${ }^{27}$. Sie befürchten, daß die Forderung nach Öffnung der Institution Ehe das Ziel habe oder doch faktisch darauf hinauslaufe, den herkömmlichen Sinn der Ehe aufzuheben oder zum Gegenstand der freien Selbstbestimmung zu machen ${ }^{28}$. Das aber würde nicht nur dem Eheverständnis, wie es die evangelischen Kirchen lehren und vertreten, widersprechen, sondern auch der ursprünglichen Absicht des Verfassungsgebers, der den grundrechtlichen Schutz der Ehe auf das in Europa über Jahrhunderte gewachsene Institut der Ehe bezogen wissen wollen. Insofern wäre eine Öffnung abzulehnen.

Demgegenüber denke ich, daß man zuerst prüfen soll, was homophile Menschen selbst erstreben, wenn sie eine Ehe schließen wollen. Die Tatsache, daß sie wirklich die Ehe im Sinne des Artikels 6 GG der Bundesrepublik Deutschland oder des Artikels 54 BV der Schweiz begehren, muß dabei in jedem Falle respektiert werden und sollte nicht dadurch diskreditiert werden, daß ganz andere Motive und Absichten vermutet werden, solange es dafür keine eindeutigen Anzeichen gibt. Sind die so plädierenden Homophilen Glieder einer evangelischen Kirche und teilen sie deren Eheverständnis, wie ich es oben als eine mögliche Auslegung ganz knapp skizziert habe ${ }^{29}$, dann wird man urteilen müssen: es geht auch hier um den lebenslangen Versuch, die eigene Leiblichkeit mit der eines anderen Menschen in einem gemeinsamen Lebensentwurf, im `Ernstfalk und also in Verantwortung gegenüber Gott, zu gestalten. So gesehen, wäre die Öffnung des Instituts der Ehe gleichermaßen aus theologischen wie aus weltlich-juristischen Erwägungen heraus geboten.

\section{Grenzen der Gleichstellung}

Die einfachste Form einerÖffnung der Institution der Ehe würde darin bestehen, daß man in den Verfassungen und Gesetzen lediglich dem Wort »Ehe« die Wörter »zwischen zwei Menschen gleichen oder ungleichen Geschlechts« hinzufügt. Wenn ich für diese undifferenzierte Öffnung nicht plädiere, so unter den heutigen Bedingungen aus folgenden Erwägungen: (1) Auch das in einem Volk weithin verbreitete, kulturell gewachsene und geprägte Verständnis von Institutionen hat Anspruch auf Achtung und Anerkennung. In diesem Sinne verbindet sich im Verständnis der meisten Menschen mit dem Begriff der Ehe primär die Vorstellung der Lebens-, Wirtschafts-, Wohn- und Geschlechtsgemeinschaft von Frau und Mann, welche monogam und auf Dauer angelegt ist. Ich sehe heute keine Notwendigkeit und auch keinen wirklich 
begründeten Anspruch, diese historisch gewachsene Einrichtung und Vorstellung gleichsam per Gesetzgebung durch eine neue Definition umzuprägen ${ }^{30}$. (2) Die verschiedengeschlechtliche Lebensgemeinschaft enthält in der Regel von Natur aus die Möglichkeit in sich, durch die Zeugung eigener Kinder an der menschlichen Kreativität zur Weitergabe und Bewahrung des Lebens teilzuhaben. Dies ist nicht der einzige oder primäre Zweck der Ehe, wie christliche Kirchen lange gelehrt haben, aber ein ihr wesentlicher, immanenter Naturzweck, der freilich mittels menschlicher Selbstbestimmung verwirklicht werden soll. Hier liegt eine gleichsam von der Natur vorgezeichnete, eigentümliche Besonderheit verschiedengeschlechtlicher Verbindungen, die nicht nivelliert werden sollte; hier würde insofern die willkürlich-definitorische Gleichsetzung zweier verschiedener Tatbestände in eine Diskriminierung der Ehe umschlagen können.

\section{Ergebnis}

Mein Ergebnis ist also an dieser Stelle: überall dort, wo es in gleich- wie verschiedengeschlechtlichen Partnerschaften um wesentlich gleiche Tatbestände und Phänomene geht, muß man sie auch rechtlich gleich behandeln; wo es sich um typische und nicht in die Entscheidungsfreiheit der Menschen gestellte Sachverhalte handelt, die charakteristische Unterschiede begründen, ist Ungleichbehandlung geboten. Deshalb sollte man in allen Fragen, die auf positivem Recht beruhende Ungleichheiten betreffen, für gleichgeschlechtliche Partnerschaften Rechtsformen öffnen, die denselben Rechtsschutz gewähren wie die bürgerliche Ehe für verschiedengeschlechtliche Partner. In allen Fragen aber, die aus Gründen der Natur oder der historisch gewachsenen kulturellen Formen auf wesentliche Unterschiede hinweisen, soll man diesen ebenfalls Rechnung tragen. Insofern plädiere ich dafür, gleichgeschlechtlichen Partnern die Möglichkeit einer amtlichen Registrierung, die nicht »Ehe « genannt werden sollte, zu öffnen, welche ihnen dieselben Rechte und Rechtswirkungen zukommen läßt wie die Eingehung einer Ehe, abgesehen von bestimmten zu spezifizierenden, nicht-diskriminierenden Ausnahmen. Diesen Weg hat das Gesetz über registrierte Partnerschaften in Dänemark gewählt, das 1989 in $\mathrm{Kraft}$ getreten ist.

\section{Ausblick}

Eine derartige Lösung würde wohl bedeuten, daß die meisten familienrechtlichen Konsequenzen des geltenden Rechtes auch für homophile Partnerschaften gelten würden, also etwa das unbedingte Verbot der Bigamie, aber auch das Vermögens-, Erb- und Scheidungsrecht. Registrierte Partnerschaft bedeutet technisch die Einfügung einer neuen Kategorie parallel zur Ehe in das Familienrecht des Bürgerlichen Gesetzbuchs (Deutschland) oder des Zivilgesetzbuches (Schweiz).

Die wichtigste Differenz zwischen Ehe und registrierter Partnerschaft im neuen dänischen Familienrecht betrifft den Sachverhalt, daß das bestehende Adoptionsrecht für Ehepaare bei registrierten Partnerschaften keine Anwendung finden soll. Hier tut sich ein weites Feld schwieriger Fragen auf, die jetzt nicht näher erörtert werden können. Ich deute nur an, in 
welcher Richtung ich weiterfragen möchte: Erstens meine ich, daß man vor jeder rechtlichen Neuordnung sorgfältig durch geeignete Forschungen ermitteln muß, wie sich eine gleichgeschlechtliche Lebensgemeinschaft auf die Entwicklung von Kindern auswirkt. Da man bei gleichgeschlechtlichen Paaren von Frauen ohnehin nicht die Verwirklichung eines bestehenden Kinderwunsches verhindern, sondern allenfalls erschweren kann, und da bei gleichgeschlechtlichen Paaren, deren einer oder beide Partner zuvor in einer heterosexuellen Beziehung mit Kindern gelebt haben können, ohnehin schwierige Fragen des Sorgerechtes entschieden werden müssen, ist hier die Frage eines denkbaren Adoptionsrechtes für männliche Paare vorrangig klärungsbedürftig ${ }^{31}$. Nach meinem heutigen Kenntnisstand plädiere ich in dieser Hinsicht gegen eine Zulassung der Adoption, weil es erstens nicht im natürlichen Vermögen von Männern allein liegt, Leben zeugend und gebärend weiterzugeben, und weil zweitens möglicherweise Knaben eines besonderen Schutzes ihrer sexuellen Selbstbestimmung bedürfen ${ }^{32}$. Auf jeden Fall aber greift in dieser ganzen Frage der Adoption ein klassisches ethisches Prinzip, das des sogenannten Tutiorismus: es besagt, daß bei Innovationen mit unsicherem Ausgang und einem nicht unerheblichen Gefahrenpotential die Beweislast für die Unschädlichkeit der geplanten Änderung beim Antragsteller liegt. Ich denke, daß hier keine ungerechtfertigte Ungleichbehandlung liegt, sondern lediglich einem Schutzbedürfnis Rechnung getragen wird.

\section{Einige Folgerungen im Blick auf kirchliche und staatliche Ordnungen}

Eine theologische Erörterung von Problemen unterschiedlicher Lebensgemeinschaften sollte wohl zuerst über die innerkirchlichen Konsequenzen ihrer Thesen und Positionen handeln, bevor sie sich - gleichsam nach außen - an Kräfte in Staat und Gesellschaft wendet. Kirche und Theologie wären nach meiner Überzeugung gut beraten, wenn sie den Ausgangspunkt ihrer Beratungen nicht bei der Homosexualität nehmen würden, sondern bei der Frage, wie Menschen mit homophiler Orientierung diese besondere Gestalt ihrer Liebe so verwirklichen können, daß sie, soweit dies Menschen zuteil werden kann, glücklich leben und von diesem Glück auch anderen mitteilen können. In dieser Perspektive ist die Frage nach den Ursachen der Homosexualität gänzlich belanglos - es gibt sie, und alle, nicht nur die homophilen Menschen, müssen mit diesem Phänomen wie mit der Gabe der Sexualität überhaupt so umzugehen lernen, daß sie einander nicht verletzen, sich nichts vorenthalten, sondern sich wechselseitig zu beschenken lernen.

\section{Anerkennung und Schutz}

Aus den bisherigen Erwägungen folgt in erster Annäherung, daß wie jede Art von Liebe unter Menschen auch die Liebe von gleichgeschlechtlichen Partnern des Schutzes, der Fürsorge und der Achtung bedarf, um frei und wechselseitig verantwortlich ihre eigenen Ausdrucksformen zu finden. Wir haben in der theologischen Erörterung dieser Fragen in den letzten vierzig Jahren dramatische Übergänge in der Beurteilung der Homosexualität erlebt: vom Laster über die Krankheit zur Abnormität und schließlich zur Andersheit. Alle Wahrnehmungen 
erweisen sich im Rückblick als Ausdruck der jeweils herrschenden gesellschaftlichen Erwartungen und Vorurteilsstrukturen. Dies gilt auch für eine liberale Haltung der Toleranz, und es ist keineswegs sicher, daß nicht auch einmal neue Diskriminierungen von Homosexuellen sich verbreiten können. Der hier gewählte Ansatz ist natürlich auch ein Kind unserer Zeit, aber ich denke, der Ausgangspunkt bei der wechselseitigen Anerkennung, Liebe und Fürsorge zweier Menschen entspricht dem biblischen Gesamtzeugnis besser als jede Fixierung auf bestimmte Ausdrucksformen der Sexualität.

\section{Kirchlich-liturgische Gestaltung}

Wenn ein Staat neue Rechtsformen für eheähnliche Partnerschaften einführt, und wenn und soweit eine Kirche die Ehe genauso wie eine neue rechtliche Form einer registrierten Partnerschaft als institutionelle Gestaltung einer verbindlichen, monogamen, auf Dauer und Treue angelegten Partnerschaft versteht, wird man fragen müssen, ob es möglich und notwendig ist, dafür auch entsprechende liturgische Formen zu finden. Nimmt man zugleich auch die Differenzen zwischen beiden Lebensgemeinschaften ernst, auf die ich verwiesen habe, dann kann es sich sinnvollerweise nicht um identische liturgische Formulare kirchlicher Vollzüge in beiden Fällen handeln, sondern es muß versucht werden, dem besonderen Charakter der jeweiligen Partnerschaft Rechnung zu tragen. Ich kann verstehen, daß homophile Menschen schon jetzt, also vor einer Neuordnung im staatlichen Familienrecht, wenigstens kirchliche Segenshandlungen erbitten, aber ein Segen - es wird heute bekanntlich viel und vieles gesegnet - kann zweifellos nicht mit einer Trauung gleichgesetzt werden. Überdies scheint es mir im Blick auf die Gebote ökumenischer Kommunikation und Rücksichtnahme geraten zu sein, daß eine Partikularkirche hier nicht einseitig vorgehen sollte ${ }^{33}$. Zur Prüfung, Erprobung und Einführung entsprechender liturgischer Formen für die Trauung gleichgeschlechtlicher Paare bedarf es insofern noch gründlicher Beratungen der kirchlichen Entscheidungsgremien mit den Praktischen Theologen und besonders den Liturgikern, wobei gewiß von vorhandenen Materialien ausgegangen werden könnte ${ }^{34}$.

\section{Homophile Partnerschaft im Pfarrhaus}

Mein Plädoyer für registrierte Partnerschaften schließt die Konsequenz ein, daß, wie verschiedengeschlechtliche Pfarrerinnen und Pfarrer mit einem Lebenspartner die Ehe eingehen können, auch gleichgeschlechtliche Paare, die ein kirchliches Amt innehaben, eine amtliche Registrierung beantragen können und, wenn die Kirche das allgemein einführen sollte, auch in besonderen liturgischen Formen getraut werden sollen. Das bedeutet zweifellos für sehr viele Kirchengemeinden eine unter heutigen Bedingungen als kaum erträglich empfundene Zumutung, und man muß deshalb mit dieser - wie mir scheint: zwingenden Konsequenz behutsam umzugehen versuchen, zumal in dieser Frage zweifellos erheblicher Sprengstoff für kirchliche Trennungen und Spaltungen liegt. Andererseits gilt aber besonders in Bereichen, in denen die Kirchen in früheren Jahrhunderten erhebliche Menschengruppen 
vielfach diskriminiert und teilweise bis zum Tode verfolgt haben, daß sie eine Pflicht zu besonderer Rücksichtnahme auf Minderheiten haben. Eine Sondermoral für Amtsträger und Amtsträgerinnen kann und darf es überdies in evangelischen Kirchen nicht geben. Andernfalls verstrickt man sich in unlösbaren Schwierigkeiten, wie sie jüngst Trutz Rendtorff nicht gescheut hat, indem er folgendermaßen argumentierte: »Eine homosexuelle Neigung ist als solche kein Hemmnis zur Ausübung des Pfarramtes, aber die offene Praktizierung homosexueller Partnerschaft, ganz zu schweigen von deren Proklamation, ist mit dem Amt eines Geistlichen, der gemäß der Ordnung der Kirche für die Glaubwürdigkeit der Verkündigung in Lehre und Unterricht wie in deren liturgischer Praxis einzustehen hat, prinzipiell unvereinbar. « ${ }^{35}$ Ich denke, daß diese merkwürdige Kombination von Toleranz und Diskriminierung psychisch und sozial unerträglich, ethisch bedenklich und auf Dauer von den Kirchen praktisch gar nicht durchzuhalten ist. Rendtorffs Argumentation läuft meines Erachtens zwingend auf eine doppelte Moral hinaus, die die homophile Neigung toleriert, hre sichtbare Gestaltwerdung in einer offiziellen Partnerschaft aber verbieten will, und die damit - wenn nicht der Absicht nach, so doch im Ergebnis - die Abspaltung und Verdrängung einer tiefen individuellen Prägung von der Person normativ verbindlich machen will. Ich halte das für die Betroffenen nicht weniger als für die Kirchen für runiös ${ }^{36}$. Im übrigen würden es die evangelischen Kirchen mit großer Wahrscheinlichkeit ohnehin äußerst schwer haben, eine vom Staat als neue Rechtsform beschlossene registrierte Partnerschaft ihren Mitgliedern - und dann auch folgerichtig ihren Amtsträgern - vorzuenthalten oder gar zu untersagen.

\section{Aufgabe einer einladenden und nicht ausgrenzenden Ehemoral}

Demgegenüber könnte die kreative Aufgabe kirchlicher Verkündigung und Ordnung gerade darin liegen, ein systematisch-theologisches Verständnis der Ehe wie der registrierten Partnerschaft - der Ausdruck ist gewiß nicht sehr glücklich! - darzulegen, welches dazu animiert, es als Beschreibung der institutionellen Chance einer lebenslänglichen Gemeinschaft zweier Menschen anzunehmen. Mir scheint, da $B$ es in den großen Kirchen schon seit langem an einer theologischen Lehre über Sexualität, Partnerschaft und Ehe mangelt, die das Ghetto der moralisierenden Betrachtung hinter sich läßt, obwohl es doch viele gute Ansätze in dieser Richtung seit Jahrzehnten gibt (z. B. bei S. Keil, St. Pfürtner, H. Ringeling), die aber nur selten in den alltäglichen innerkirchlichen Konflikten wirklich rezipiert werden.

Gar nicht vermag ich deshalb vor dem Hintergrund meiner Überlegungen zu akzeptieren, wenn homophile Partner nunmehr auf die Möglichkeit offener Beziehungen, sogenannter nichtehelicher Lebensgemeinschaften hingewiesen werden, welche ihrerseits ja wiederum längst erheblichen Verrechtlichungen unterliegen. Denn während verschiedengeschlechtliche Partner in dieser Hinsicht eine Wahlfreiheit haben, ist dies bei gleichgeschlechtlichen Partnern gerade nicht gegeben. Wenn man schließlich die vermeintliche oder tatsächliche Promiskuität homosexueller Menschen, angeblich besonders der Männer, beklagt und daraus folgert, daß der Ehe nahekommende Rechtsformen für sie nicht statthaft sein dürfen, verstrickt man sich in einen Widerspruch, wenn man ausgerechnet in diesem Falle rechtliche Ordnungen, die auf Dauerhaftigkeit und Vorbehaltlosigkeit einer Beziehung setzen, ablehnt. 


\section{Zum Verhältnis Zivilehe - kirchliche Trauung}

Nicht akzeptabel erscheinen mir einige neuerdings in der Schweiz erwogene Vorschläge, die darauf hinauslaufen, daß die staatlich garantierte Zivilehe ausschließlich verschiedengeschlechtlichen Partnern vorbehalten bleiben soll, während gleichzeit der Kirche das Recht zugebilligt werden könne, ihrerseits Verbindungen nach eigenem Recht anzuerkennen, vielleicht sogar zu begründen und liturgisch mit Segenshandlungen zu begleiten. Obwohl es Staaten gibt, in denen weltliche und kirchliche Ordnungen der Ehe nebeneinander existieren und die Zivilehe nicht obligatorisch vorgeschrieben ist wie in Deutschland oder in der Schweiz ${ }^{37}$, vermag ich in der Einführung derartiger Regelungen keinen rechtspolitischen Fortschritt zu sehen. Der Grund dafür liegt einfach darin, daß die Zivilehe mit ihrer relativ klaren Definition von Rechten und Pflichten der Partner sowie der Angehörigen gerade für die schwächeren Partner einen unerläßlichen Schutz bietet, insbesondere im Scheidungsfall. Dieser Schutz aber, so befürchte ich, könnte bei einer Wahlfreiheit zwischen verschiedenen EheschlieBungsformen staatlichen wie kirchlichen Eherechtes nur zu leicht auf der Strecke bleiben. Dabei ist auch zu bedenken, daß in einer multireligiösen Gesellschaft gerade die Konfliktnormen sinnvollerweise strikt weltlicher Art sein müssen, wenn man den interkonfessionellen wie interkulturellen Religionsfrieden erhalten will. Im übrigen wäre es vermutlich auch gar nicht zu vermeiden, für Ehen, die nach dem partikularen Recht einer anerkannten Religionsgemeinschaft geschlossen werden dürften, erneut eine gewisse staatliche Genehmigung vorzuschreiben, denn der Rechtsstaat zieht seine innere Legitimität nicht zuletzt aus der Tatsache, daß er im Zweifel um willen der Menschenwürde sogar religiöse Selbstbestimmungsansprüche einschränken muß, wenn deren Folgen die Freiheit und Würde anderer Menschen verletzen könnten.

\section{Zusammenfassung}

Das staatliche Recht leidet durch die Einführung registrierter Partnerschaften keinerlei Einbußen. Im Gegenteil: so wie Staat und Gesellschaft in Dänemark nicht zuletzt aus gesundheitspolitischen Gründen die neue Rechtsform bejaht haben, kann die Gesellschaft aus vielerlei Gründen eigentlich nur ein starkes Interesse daran haben, daß homophile Partnerschaften so stabil wie möglich sind. Deshalb sollten sie eines ähnlichen Schutzes teilhaftig werden, wie ihn der Staat von Verfassungs wegen der Ehe verliehen hat. Die Ehe und ihre Würde werden dadurch nach meiner Einsicht nicht geschmälert, sondern eher gefördert. Daß aber die Institution der bürgerlichen Ehe Bestand hat, hängt nicht zuletzt auch davon $a b$, ob ihre christlich-theologische Deutung als Ernstfall der Liebe auch in einer säkularen Gesellschaft von den Menschen als Beschreibung einer überzeugenden, befreienden und verpflichtenden Lebensform erfahren und bejaht werden kann.

\section{Prof. Dr. Wolfgang Lienemann}

\section{Universität Bern}

Pilgerstraße $35-\mathrm{CH}-4055$ Bern 
The author pleads for an equal and differentiated treatment of marriage (between woman and man) and same-sex-unions (between gay people) in civil law. In theological perspective it is necessary to regard same-sex-unions as well as marriages under the aspects of life-long commitment and responsibility - both may be and should be »love in earnest « ( $»$ Liebe im Ernstfall - K. Barth) For the decisive moment in every kind of life-long partnership in the destination that "your body is a temple of the Holy Spirit " $(1$ Kor 6,19). - From this central point of view biblical damnations of homosexual behaviour must be criticised. - As further consequences the articel discusses the legal introduction of the possibility of »registered partnership« for gay people and following options in liturgy and law of the church.

\section{Anmerkungen}

1. Anlaß war eine Tagung der Ehekommission der Ev.-Ref. Kirchen Bern - Juta zum Thema "Ehe und Familie für alle? Zur Gleichstellung homosexuell liebender Frauen und Männer in Ehe und Familie« am 13.1. 1995 in Bern. Vorgetragen wurden die Abschnitte I und II; die Ausführungen sind hier um Abschnitt III sowie die nötigsten Nachweise ergänzt.

2. Aus der Literatur vgl. besonders $H$. Thielicke: Theologische Ethik III, Tübingen 1964, 788-810; H. G. Wiedemann: Homosexuelle Liebe. Für eine Neuorientierung in der christlichen Ethik, Stuttgart - Bern 1982; H. Kentler (Hg.): Die Menschlichkeit der Sexualität. Berichte - Analysen - Kommentare ausgelöst durch die Frage: Wie homosexuell dürfen Pfarrer sein?, München 1983; H. Ringeling: Homosexualität: Erscheinung und Bewrtung, ZEE 31, 1987, 6-35 und $82-102$, wieder abgedruckt in: ders.: Christliche Ethik im Dialog. Beiträge zur Fundamental- und Lebensethik II, Freiburg/Ue. - Wien 1991, 163-212; ;B. Kittelberger/W. Schürger/W. Heilig-Achneck (Hgg.): Was auf dem Spiel steht. Diskussionsbeiträge zu Homosexualität und Kirche, München 1993. - Die hier vertretene Auffassung berührt sich am stärksten mit Erwägungen von W. Joest: Verletzte Liebe?, in: Kittelberger, u. a.: 286-296.

3. Vgl. besonders die Stellungnahmen: Homosexuelle Liebe, hg. von der Ev. Kirche im Rheinland - Landessynode 1992, Düsseldorf 1992; sowie: Homosexuelle in der Kirche? Ein Text der Theologischen Studienabteilung beim Bund der Evangelischen Kirchen in der DDR, hg. von der Aktion Sühnezeichen/Friedensdienste, Berlin 1985.

4. In Ermangelung eines besser geeigneten Ausdrucks verwende ich diese unschöne Bezeichnung, die m. W. zum ersten Mal in dem entsprechenden dänischen Gesetz von 1989 begegnet (s. unten bei Anm. 19). Das Europaparlament verwendet den Ausdruck »eingetragene Partnerschaft«; vgl. den Bericht des Ausschusses für Grundfreiheiten und innere Angelegenheiten über die Gleichberechtigung von Schwulen und Lesben in der EG v. 26. 1. 1994 (DOC-DE/RR/244267).

5. Vgl. außer den Ausführungen bei Ringeling (Anm. 2) bes. G. Strecker: Homosexualität in biblischer Sicht, KuD 28, 1982, 127-141; P. von der Osten-Sacken: Paulinisches Evangelium und Homosexualität, BThZ 3, 1986, 28-49; J. Becker:Zum Problem der Homosexualität in der Bibel, ZEE 31, 1987, 36-59; H. Balz: Biblische Aussagen zur Homosexualität, ebd., 60-72; K. Wensgt: Paulus und die Homosexualität, ebd. 72-81; W. Stegemann: Keine ewige Wahrheit. Die Beurteilung der Homosexualität bei Paulus, in: Kittelbeger u. a.: Was auf dem Spielt steht, 262-285; E. Stegemann, Antihomosexualität bei Paulus, in: H. Puff (Hg.): Lust, Angst und Provokation. Homosexualität in der Gesellschaft, Göttingen/Zürich 1993, 67-78; sowie die Ausführungen in: Homosexuelle Liebe, 20-24. Dazu wiederum kritisch $K$. Haacker: Exegetische Gesichtspunkte zum Thema Homosexualität, in: Theol. Beiträge 25, 1994, 173-180.

6. Vgl. E. S. Gerstenberger: Das Buch Mose. Leviticus (ATD 6), Göttingen 1993, 224-232 und 270-275.

7. Vgl. dazu besonders die Ausführungen bei Ringeling: aaO.

8. Zum folgenden vgl. bes. W. Neidhart: Das paulinische Verständnis der Liebe und die Sexualität, ThZ 40 (1984), 245-256.

9. Das Elend des Christentums oder Plädoyer für eine Humanität ohne Gott, Reinbek 1968, 50, zit. bei Neidhart: 246.

10. Vgl. P. Brown: The Body and Society. Men, Women and Sexual Renunciation in Early Christianity, New York 1988 (deutsch: Die Keuschheit der Engel. Sexuelle Entsagung, Askese und Körperlichkeit im frühen Christentum, München - Wien 1991, TB München 1994); E. Pagels: Adam, Eve and the Serpent, New York 1988 (deutsch: Adam, Eva und die Schlange. Die Geschichte der Sünde, Reinbek 1991, TB 1994).

11. Neidhart: aaO., 250.

12. Ebenda, 254. 
13. Vgl. zu diesen paulinischen Ausführungen neuderdings $R$. Kirchhoff: Die Sünde gegen den eigenen Leib. Studien zu porné und porneia und dem soziokulturellen Kontext der paulinischen Adressaten, Göttingen 1994.

14. Vgl. beispielsweise $M$. Barz/H. Leistner/U. Wild: Hättest du gedacht, daß wir so viele sind? Lesbische Frauen in der Kirche, Stuttgart 1987; B. Kittelberger aaO.; Th. Grossmann: Eine Liebe wie jede andere. Mit homosexuellen Jugendlichen leben und umgehen, Reinbek 1993.

15. Zum verfassungsrechtlichen Verständnis der Gleichheit als Willkürverbot und Gestaltungsauftrag vgl. $R$. Herzog: Art. Gleichheit II. Gleichheit in der Staatstheorie, Ev. Staatslexikon 3. Aufl., Stuttgart 1987, Bd. 1, 1182-1190 (Lit.).

16. Zu Luthers Eheauffassung vgl. jetzt speziell im Blick auf das »Traubüchlein" (WA 30 III, 43-80), aber mit einer großen Fülle weiterer Belege A. Peters: Kommentar zu Luthers Katechismen, Bd. 5: Beichte, Haustafel, Traubüchlein, Taufbüchlein, hg. v. G. Seebaß, Göttingen 1994, 119-155.

17. Vgl. W.-D. Marsch: Art. Institution, RGG 3. Aufl., Bd. 3 (1959), 783-785; H. E. Tödt: Art. Institution, TRE 16 (1987), 206-220.

18. E. Wolf: Sozialethik. Theologische Grundfragen, hg. v. Th. Strohm, Göttingen 1975, 173.

19. Die Kirchliche Dogmatik III/4, 209, $16 \mathrm{ff}$.

20. Vgl. dazu U.P. Jauch: Immanuel Kant zur Geschlechterdifferenz. Aufklärerische Vorurteilskritik und bürgerliche Geschlechtsvormundschaft, Wien 1988; S. Kofman: Die Ökonomie der Achtung. Kant, in: H. Nagl-Docekal (Hg.): Feministische Philosophie, Wien - München 1990, 41-62.

21. Den dänischen Gesetzestext mit einem kurzen Kommentar findet man bei A. Wacke: Die Registrierung homosexueller Partnerschaften in Dänemark, FamRZ 1990, Heft 4, 347-351. Einen vergleichenden Überblick gibt H. Thofern: Entwicklungstendenzen des Familienrechts im Ausland, FuR 1992, 342-346; zu einem nicht unwichtigen Spezialproblem vgl. A. Merschmeier: Zur Frage des Eintritts des überlebenden Partners einer gleichgeschlechtlichen Lebensgemeinschaft in den Mietvertrag bei Tod des Mieters, ZMR 1994, 13-15. - Für Hinweise auf neuere juristische Literatur in Deutschland danke ich Marion und Rainer Eckertz-Höfer..

22. Zu den vielfachen Ungleichheiten und Diskriminierungen der Partner in gleichgeschlechtlichen Lebensgemeinschaften in der Schweiz vgl. A. Geissbühler-Blaser: Welche rechtlichen Veränderungen sind in der Diskussion? Welche Ungleichheiten gibt es? (Manuskript Januar 1995); zur Situation in Deutschland vgl. M. Bruns: Homosexuelle im Recht: Toleriert, aber nicht akzeptiert, Betrifft JUSTIZ Nr. 34 (Juni 1993), 82-86.

23. M. Bruns/V. Beck: Das Eheverbot bei Gleichgeschlechtlichkeit, MDR 9/1991, 832-835 (834). Demgegenüber hat das BVerfG 1993 eine Verfassungsbeschwerde eines gleichgeschlechtlichen Paares wegen Verletzung der Eheschließungsfreiheit nicht angenommen, u. a. mit der Begründung: »Mangels erkennbarer Anhaltspunkte für einen grundlegenden Wandel des Eheverständnisses in dem Sinne, daß der Geschlechtsverschiedenheit der Ehepartner keine prägende Bedeutung zukommt, besteht kein Anlaß, von der durch die ständige Rechtsprechung des BVerfG bestätigten Beschränkung des Schutzbereiches von GG Artikel 6 Absatz 1 auf die Lebensgemeinschaft von Mann und Frau abzuweichen. «(Az. 1 BvR 640/93) Es wird in den einschlägigen Urteilen mit der Nominaldefinition der Ehe als einer "Lebensgemeinschaft eines Mannes mit einer Frau« dahingehend argumentiert, daß schon begriffslogisch eine Ehe zwischen gleichgeschlechtlichen Partnern wunmöglich« sei. Das BVerfG hat aber deutlich gezeigt, inwiefern diese Argumentation auf Grenzen stoßen kann, nämlich dann, wenn ein erkennbarer Wandel im Eheverständnis vorliegt.

24. Vgl. zuletzt $H$. Ringeling: Homosexualität als Frage kirchlichen Handelns, ZEE 38, $1994,163-167$.

25. Christliche Ethik im Dialog (Anm. 2): bes. 204-211.

26. Ringeling bezieht sich hier (ebenda, 205) auf St. Pfürtner: Sexualität und Kirche, Hamburg $1972,256$.

27. Selbstbestimmung und Institution. Ethisch-theologische Implikationen der Kontroverse um »Homosexualität und Pfarrerberuf «, ZEE 38, 1994, 190-202.

28. Rendtorff behauptet pauschal im Blick auf die Homosexualität: »Die Konkretion von Selbstbestimmung als dem Handlungskern von Menschenwürde und Menschenrecht verläuft über Sexualität. Sexualität erscheint dabei als Inbegriff des individuellen Selbstverständnisses. « (192) Und weiter: »Selbstbestimmung als Selbstverwirklichung durch Sexualität, das ist die Grundformel, mit der die Anerkennung der Homosexualität sich mit der Kritik an der Ehe als gesellschaftlicher Konvention verbindet.« (193) Abgesehen davon, daß eine derartige Bedeutung der Sexualität auch bei heterosexuell geprägten Menschen begegnet (man erinnere sich an W. Reich: Massenpsychologie des Faschismus, 1933), ist die Verallgemeinerung (»Inbegriff des Selbstverständnisses«, »Grundformel«) überhaupt nicht durch Selbstzeugnisse homophiler Menschen gedeckt und insofern diskriminierend. Vollends fatal erscheint mir die Behauptung, daß eine homophile Neigung als solche zwar keine "Sünde« sei, wohl aber »der allgemein gesetzte Wille, sich in der Beziehung zu Gott (?? WL) und zur Gemeinde durch Homosexualität zu definieren, zur Infragestellung der Gemeinschaft « führe (193f.) Ich verstehe diese etwas merkwürdigen Formulierungen so, da $B$ nach Rendtorff die homophile Neigung toleriert werden muß, aber der Anspruch auf Anerkennung zurückgewiesen werden soll. Homophilen Menschen wird auf diese Weise eine Sexualitätsfixierung unterstellt, die gerade nicht im 
Zentrum ihrer ausdrücklichen Forderungen nach Eheschließung steht; an die Stelle authentischer Äußerungen homophiler Menschen tritt ein Bild, das ihre weitere Diskriminierung zu legitimieren geeignet ist. Tatsächlich wollen aber nach meiner Kenntnis homophile Menschen nicht im besonderen Bezug auf ihre Form der Sexualität Anerkennung finden, sondern in dieser Hinsicht lediglich sowenig wie alle anderen Menschen auch benachteiligt werden. Die Bedeutung des authentischen (Glaubens-)Zeugnisses homophiler Menschen betont dagegen zurecht B. Wannenwetsch: Das "Natürliche« und die »Moral«. Zur neueren Diskussion um die Homosexualität in der Kirche, ZEE 38, 1994, 168-189.

29. Vgl. dazu zuletzt B. Wannenwetsch: Die Freiheit der Ehe. Das Zusammenleben von Frau und Mann in der Wahrnehmung evangelischer Ethik, Neukirchen - Vluyn 1993.

30. So auch im Kern das deutsche BVerfG. Diese Position schließt aber die Möglichkeit ein, daß sich genau diese gesellschaftlich verbreitete Auffassung im Laufe der Zeit ändern kann, wenngleich derzeit dafür kaum empirische Anzeichen zu entdecken sind.

31. Allerdings halte ich angesichts der tatsächlichen Probleme von Adoptionen in der Schweiz und in Deutschland dies für eine ausgesprochen »akademische" Frage, denn die Adoptionshindernisse sind in der Regel so groß, daß sich derartige Fragen in der Praxis kaum stellen dürften.

32. Vgl. dazu die bedenkenswerten Überlegungen von S. Tönnies: Symbolische Gesetzgebung: Zum Beispiel § $175 \mathrm{StGB}$, ZRP 1992, 411-413.

33. Vgl. dazu meinen Beitrag zum Thema: Vf:: Partikularkirchen und ökunenische Bewegung, in: G. Rau/H.-R. Reuter/K. Schlaich (Hg.): Das Recht der Kirche, Bd. 2, Gütersloh 1995, 318-376.

34. Vgl. mit vielfachen Anregungen und Beispielen E. Stuart (ed.): Daring to Speak Love's Name. A Gay and Lesbian Prayer Book, London 1992 zu altkirchlichen und mittelalterlichen Vorjäufern vgl. bes. J. Boswell: Same-Sex-Unions in Premodern Europe, New York 1994.

35. AaO., 201.

36. Daß manche Kirchen hier erheblichen weiteren Beratungsbedarf empfinden, zeigen Urteile von Spruchkammern und Äußerungen von Synoden lutherischer Kirchen in Deutschland, wenn in Streitfällen nicht auf Entfernung aus dem Dienst, sondern lediglich auf Versetzung in den Wartestand erkannt wird, weil und sofern in den nächsten Jahren die Kirchen ihre Beurteilung gleichgeschlechtlicher Partnerschaften ändern und dann die entsprechenden kirchenrechtlichen Bestimmungen anpassen könnten; vgl. dazu B. Guntau: Homosexuelle Lebensgemeinschaft im Pfarrhaus. Anmerkungen zum Beschluß der Synode der Ev.-luth. Landeskirche Hannovers vom 24. November 1993, ZevKR 39, $1994,138-160$.

37. Die internationale Vielfalt berücksichtigt W. Müller-Freienfels: Art. Ehe, Entscheidung 1. Rechtlich, EKL 3. Aufl, Bd. 1 (1986), 956-965. 